\title{
Characterization of PRLR and PPARGC1A genes in buffalo (Bubalus bubalis)
}

\author{
Ruheena Javed $^{1,2}$, Sanjeev K. Gautam ${ }^{2}$, Ramesh K. Vijh ${ }^{1}$ and Madhu S. Tantia ${ }^{1}$ \\ ${ }^{I}$ National Bureau of Animal Genetic Resources, Karnal, Haryana, India. \\ ${ }^{2}$ Department of Animal Biotechnology, Kurukshetra University, Kurukshetra, Haryana, India.
}

\begin{abstract}
More than 40 million households in India depend at least partially on livestock production. Buffaloes are one of the major milk producers in India. The prolactin receptor (PRLR) gene and peroxisome proliferators activated receptor- $\gamma$ coactivator 1-alpha (PPARGC1A) gene are reportedly associated with milk protein and milk fat yields in Bos taurus. In this study, we sequenced the PRLR and PPARGC1A genes in the water buffalo Bubalus bubalis. The PRLR and PPARGC1A genes coded for 581 and 819 amino acids, respectively. The $B$. bubalis PRLR gene differed from the corresponding Bos taurus at 21 positions and four differences with an additional arginine at position 620 in the PPARGC1A gene were found in the amino acid sequence. All of the changes were confirmed by cDNA sequencing. Twelve buffalo-specific single nucleotide polymorphisms (SNPs) were identified in both genes, with five of them being non-synonymous.
\end{abstract}

Key words: Bubalus bubalis, buffalo, PRLR gene, PPARGC1A gene.

Received: February 1, 2011; Accepted: June 7, 2011.

The water buffalo (Bubalus bubalis) is an important dairy animal in the Indian subcontinent, with $55 \%$ of the milk in India being produced by buffaloes. With over 105 million buffaloes (56\% of the world's total population), India needs to improve the productivity of buffaloes as this species is rapidly replacing cattle in several milk-producing areas. Many candidate genes have been associated with dairy performance traits. The prolactin receptor (PRLR) gene, which belongs to the hematopoietin receptor superfamily (Kossiakoff et al., 1994), is particularly promising in this regard because of its crucial role in signal transduction from lactogenic hormones to milk protein gene promoters (Hayes et al., 1996). Prolactin (PRL) regulates milk gene expression (Gao et al., 1996) by activating Janus kinase 2 (JAK2) and signal transducer and activator of transcription 5 (STAT5), initially termed mammary gland factor (MGF). The gene coding for bovine PRLR was mapped to chromosome 20 in cattle and chromosome 19 in buffalo (Amaral et al., 2008). This gene has nine exons that code for a polypeptide of 581 amino acids.

The bovine peroxisome proliferators activated receptor- $\gamma$ coactivator 1 -alpha (PPARGC1A) gene is another putative gene associated with a previously described quantitative trait locus (QTL) for milk fat yield on BTA6 (Weikard et al., 2005). The protein encoded by this gene is a transcriptional coactivator that regulates the genes involved in

Send correspondence to Madhu S. Tantia. DNA Sequencing Lab, National Bureau of Animal Genetic Resources, Karnal, 132001 Haryana, India. E-mail: tantiams@gmail.com. energy metabolism. The bovine PPARGC1A gene is organized into 14 exons consisting of $6261 \mathrm{bp}$ and is expressed at different levels in a large number of tissues.

In this work, we determined the exon sequences of the buffalo PRLR and PPARGC1A genes and confirmed the exon-intron boundaries by cDNA sequencing. We also identified the SNPs in a panel of various buffalo breeds.

The PRLR and PPARGC1A genes were characterized in a panel of 24 animals drawn from six Indian water buffalo breeds (Murrah, Bhadawari, Tarai, Pandharpuri, Marathwada and Mehsana). The Murrah breed is a northern Indian large dairy breed whereas Bhadawari and Tarai are small sized breeds adapted to extensive production systems. The Pandharpuri and Marathwada breeds are medium sized buffaloes from central India and the Mehsana breed is a western Indian dairy breed. DNA was isolated from blood samples and diluted to an optimum concentration. The genomic regions corresponding to the putative buffalo PRLR and PPARGC1A genes were amplified by the polymerase chain reaction (PCR) with 11 primer pairs for PRLR and 17 primer pairs for the PPARGC1A gene designed from the Bos taurus GenBank accession nos. AJ966356 and AY321517, respectively, at exon-intron boundaries (Table 1). The amplifications were run in $10 \mu \mathrm{L}$ reaction mixtures containing $50 \mathrm{ng}$ of genomic DNA, 5 pmol of each primer, $2 \mathrm{mM} \mathrm{MgCl}_{2}, 0.2 \mu \mathrm{M}$ of each dNTP and 1 unit of Taq DNA polymerase (Sigma) in a thermocycler (icycler Biorad, San Diego, CA, USA). The amplification protocol consisted of $5 \mathrm{~min}$ denaturation at $94{ }^{\circ} \mathrm{C}$ followed by 35 amplification cycles of $94^{\circ} \mathrm{C}$ for $45 \mathrm{~s}, 55^{\circ} \mathrm{C}$ 
Table 1 - Primers used in this work.

\begin{tabular}{|c|c|c|}
\hline Primer name & Forward primer & Reverse primer \\
\hline \multicolumn{3}{|l|}{ PRLR gene } \\
\hline PRL1 & TCTgTTCATggAggCAAATg & AAgCAACAgCAggACAgATg \\
\hline PRL2 & TgTgCCTCACCAgACTTTTg & gggACTgTgATggATTCTCC \\
\hline PRL3 & CCCCATCTACCTgCTTCTgT & AATTAACgCAgggTCAgTgg \\
\hline PRL4 & AgCAAggAAgCTCCATACCA & CggggATCTATCCCTAAgACA \\
\hline PRL5 & AAgCTAgCgAgATCTgCCTCT & TAggAggCACgACTggTTCT \\
\hline PRL6 & gACCTACATACTggCTTCTCTgC & AAAACAATggCAgATTTCAgg \\
\hline PRL7 & ggCAggggACTTATgTTCAA & gAggTgCTTgAATTATCTgTAggTT \\
\hline PRL8 & CATgTAgCAgCCATTTggAA & gCTTAgCCAgACTgCACTgA \\
\hline PRL9 & gCgTTCACTTTgATTgCAgA & TgTTggTCTgTTTggTCCAg \\
\hline PRL10 & CAACATTgCTgACgTgTgTg & CAATTgAACCCATCCTTCCA \\
\hline PRL11 & gTgTCCCgggTgACAgATAg & AAACCATgAAggCCTTTTCC \\
\hline \multicolumn{3}{|c|}{ PPARGC1A gene } \\
\hline PRGC_1 & CATgATgCTCCAAAAgTCCA & CCCCTCACAggAATATTTgC \\
\hline PRGC_2 & ТTTTTCTCCCTgCСТCCTg & CAAAgCAAgAACCCATTATgC \\
\hline PRGC_3 & CTCATCTCCCAgTgTCAACTCA & gTAgCCAgAggCAACTCCAA \\
\hline PRGC_5 & AAAgTTTTAgTAgCTTATTCTCATgCT & TCCAgCTgAATTAATACATAgAATCC \\
\hline PRGC_6 & TgAACACTTCATTgAAAATCTCATC & gAAgTTTgggTgTCCTCAgC \\
\hline PRGC_7 & AgTTTTCCTgTTTCCAgTTTCC & ATATACATTTCACATACACACTCATCC \\
\hline PRGC_8 & ggAAAATgTgTCCTggCATT & gCggTCTCTCTCAggTAgCA \\
\hline PRGC_9 & AggAgCTCCATgACTCCAgA & CTTAggCTTTgggTgggTTT \\
\hline PRGC_10 & TCCAggggCTACTCAgTCAT & ggCAgggTTTTggACTACAT \\
\hline PRGC_11 & gCССССТCССТAgTTTATgA & AATCTATgCCCATCACATCC \\
\hline PRGC_12 & TgCCTTCATAAATggTTCTgg & CTgTCAAAgCATTCCCATCC \\
\hline PRGC_13 & gCgAAgTCTCCAAAgTggAA & AgTAATggTTTggCCCAATg \\
\hline PRGC_14 & CggATAAATTgTTTTTgAATCg & TgCAATAgTCTTTTAgggAAggA \\
\hline PRGC_15 & gAAgACACCgCAggTTCTgTA & TTCTCgATTAAgAAAAATTTAgCAg \\
\hline PRGC_16 & TTTgTTACAgCTATgCACTgTAAATg & gCATTTTgTATCAAATTAAAATCACA \\
\hline PRGC_17 & CCATTAAgAAATgTTTTTATTTTCTCT & gAgTgTggAgCCCTggAAT \\
\hline
\end{tabular}

(variable) for $45 \mathrm{~s}$ and $72{ }^{\circ} \mathrm{C}$ for $45 \mathrm{~s}$, with a final extension at $72{ }^{\circ} \mathrm{C}$ for $10 \mathrm{~min}$. The PCR products were separated on $2 \%(\mathrm{w} / \mathrm{v})$ agarose (Promega, USA) gels to determine fragment sizes. PCR products were then incubated with exonuclease and calf intestinal phosphatase ( 1 unit each) at $37^{\circ} \mathrm{C}$ for $2 \mathrm{~h}$ followed by inactivation at $85^{\circ} \mathrm{C}$ for $15 \mathrm{~min}$ and subjected to cycle sequencing reactions in a $5 \mu \mathrm{L}$ reaction volume using the Big-Dye system (Applied Biosystems, USA) according to the manufacturer's instructions. Sequencing was done in a 3100 Avant automated sequencer (Applied Biosystems, USA) using forward and reverse primers.

Total RNA was isolated from buffalo mammary gland tissue using a High pure RNA kit (Roche, Germany). cDNA was synthesized from total RNA using a superscript reverse transcriptase system (RT-PCR) and a first strand cDNA synthesis kit (Invitrogen, New Zealand) according to the manufacturers instructions. Sequencing was done in a 3130xl Genetic Analyser (Applied Biosystems) using forward and reverse primers designed for the coding region.
The sequences obtained for each fragment were aligned with SeqScape software v.2 (Applied Biosystems) to obtain PRLR and PPARGC1A gene consensus sequences using the B. taurus sequances as reference. The predicted amino acid sequences were obtained using AnnHyb 4.943 software (Friard, 2010). Single nucleotide polymorphisms (SNPs) were detected in the buffalo PRLR and PPARGC1A genes and confirmed in additional samples.

The PRLR gene sequence of $B$. bubalis consisted of nine exons. Overall, $4338 \mathrm{bp}$ of genomic DNA (GQ339914) and 1239 bp of cDNA (HQ236497) were sequenced. The buffalo PRLR gene encoded 581 amino acids. Comparison with the corresponding $B$. taurus sequence revealed 21 amino acid differences (positions 2, 19, 52, 57, 61, 66, 100, 208, 243, 299, 338, 347, 399, 443, 480, 485, 494, 497, 534, 539 and 548). Six buffalo- specific SNPs were identified in the PRLR exon regions of the $B$. bubalis gene (positions G305T ${ }^{*}$ in exon 2, A1017G in exon 4, and A2690G*, G3009A*, A3221G and A3486G in exon 9). Three SNPs were non-synonymous $\left(^{*}\right)$, resulting in amino 
Table 2 - Buffalo-specific SNPs identified in the PRLR and PPARGC1A genes.

\begin{tabular}{|c|c|c|c|c|c|c|}
\hline Gene & Position & NCBI dbSNP accession no. & SNP & Codon change & Region & Amino acid change \\
\hline \multirow{6}{*}{$\begin{array}{l}\text { PRLR } \\
\text { (GQ339914) }\end{array}$} & 305 & SS\# 410759785 & $\mathrm{G} / \mathrm{T}^{*}$ & GTC/TTC & Exon2 & $\mathrm{V} 19 \mathrm{~F}$ \\
\hline & 1017 & SS\# 410759786 & $\mathrm{~A} / \mathrm{g}$ & & Exon4 & \\
\hline & 2690 & SS\# 410759787 & $\mathrm{~A} / \mathrm{g}^{*}$ & $\mathrm{CAC} / \mathrm{CGC}$ & & H328R \\
\hline & 3009 & SS\# 410759788 & $\mathrm{G} / \mathrm{A}^{*}$ & GGC/AGC & & G428S \\
\hline & 3221 & SS\# 410759789 & $\mathrm{~A} / \mathrm{g}$ & & Exon9 & \\
\hline & 3486 & SS\# 410759790 & $\mathrm{~A} / \mathrm{g}$ & & & \\
\hline \multirow{6}{*}{$\begin{array}{l}\text { PPARGC1A } \\
\text { (GU066311) }\end{array}$} & 718 & SS\# 410759779 & $\mathrm{C} / \mathrm{T}$ & & Intron3 & \\
\hline & 1844 & SS\# 410759780 & $\mathrm{~A} / \mathrm{g}$ & & Exon6 & \\
\hline & 1902 & SS\# 410759781 & $\mathrm{C} / \mathrm{T}$ & & Intron6 & \\
\hline & 2382 & SS\# 410759782 & $\mathrm{G} / \mathrm{T}^{*}$ & GGG/TGG & & W346G \\
\hline & 2529 & SS\# 410759783 & $\mathrm{C} / \mathrm{T}^{*}$ & $\mathrm{CTC} / \mathrm{TTC}$ & Exon8 & F395L \\
\hline & 2657 & SS\# 410759808 & $\mathrm{~A} / \mathrm{g}$ & & & \\
\hline
\end{tabular}

*Non synonymous

acid changes at positions V19F, H328R and G428S (Table 2).

The PPARGC1A gene (GU066311) was sequenced by designing primers at the exon-intron boundaries for most of the exons because the large size of the introns precluded sequencing. However, intron 6 was small enough to be completely sequenced. The amino acid sequence predicted from genomic DNA was confirmed by sequencing the cDNA (HQ236498). The PPARGC1A gene encoded 819 amino acids and comparison with the corresponding $B$. taurus sequence revealed four differences (positions 392, 419, 534 and 774), with the additional amino acid (arginine) at position 620. Six buffalo-specific SNPs were identified in the PPARGC1A gene (positions C718T in intron 3, A1844G in exon 6, C1902T in intron 6, and G2382T*,

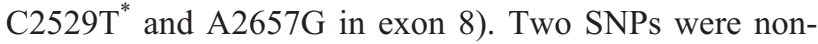
synonymous $(*)$, resulting in amino acid changes at positions W346G and F395L (Table 2).

Comparison with the corresponding B. taurus genes revealed that in both species the two genes contained an equal number of exons of similar length, except for exon 9 in the PPARGC1A gene, which had three more nucleotides that encoded an additional amino acid (arginine). The sequence conservation and exon-intron splice sites of $B$. bubalis and $B$. taurus were confirmed by sequencing the cDNA of both genes.

In this study, we sequenced and characterized the PRLR and PPARGC1A genes that are putative candidate genes for milk quality and production in buffaloes. We also identified novel buffalo-specific SNPs. Of the 12 SNPs identified, five were non-synonymous, i.e., they can theoretically change protein structure and function. These SNPs may be useful as markers for milk quality and production traits in buffaloes.

\section{Acknowledgments}

The authors gratefully acknowledge the financial assistance from National Agricultural Innovation Project
C1050 of Indian Council of Agricultural Research, New Delhi.

\section{References}

Amaral MEJ, Grant JR, Riggs PK, Stafuzza NB, Filho EA, Goldammer T, Weikard R, Brunner RM, Kochan KJ, Greco AJ et al. (2008) A first generation whole genome RH map of the river buffalo with comparison to domestic cattle. BMC Genomics 9:631.

Gao J, Hughes JP, Auperin B, Buteau H, Edery M, Zhuang H, Wojchowski DM and Horseman ND (1996) Interactions among Janus kinases and the prolactin (PRL) receptor in the regulation of a PRL response element. Mol Endocrinol 10:847-856.

Hayes H, Le CC, Goubin G, Mercier D, Payen EJ, Bignon C and Kohno K (1996) Localization of ZNF164, ZNF146, GGTA1, SOX2, PRLR and EEF2 on homeologous cattle, sheep, and goat chromosomes by fluorescent in situ hybridization and comparison with human gene map. Cytogenet Cell Genet 72:342-346.

Kossiakoff AA, Somers W, Ultsch M, Andow K, Muller YA and De Vos AM (1994) Comparison of the intermediate complexes of human growth hormone bound to the human growth hormone and prolactin receptors. Prot Sci 3:16971705.

Weikard R, Kuhn C, Goldammer T, Freyer G and Schwerin M (2005) The bovine PPARGC1A gene: Molecular characterization and association of an SNP with variation of milk fat synthesis. Physiol Genom 21:1-3.

\section{Internet Resources}

Friard O (2010) AnnHyb 4.943 Beta: Center for Molecular Systems Biology, University of Torino, Via Accademia Albertina, http://www.bioinformatics.org/annhyb/download.php (October 7, 2010).

Associate Editor: Alexandre Rodrigues Caetano

License information: This is an open-access article distributed under the terms of the Creative Commons Attribution License, which permits unrestricted use, distribution, and reproduction in any medium, provided the original work is properly cited. 